\title{
THE FREE ORTHOMODULAR WORD PROBLEM IS SOLVABLE
}

\author{
GUDRUN KALMBACH
}

It is shown that the free orthomodular word problem is solvable. Since the free orthomodular lattice $L_{O}$ on countably many generators has, as a partial subalgebra, every finite partial orthomodular lattice $P$, which is contained in some orthomodular lattice as a partial subalgebra, it is sufficient to prove Evans embedding property for these $P$ only. The construction of the finite orthomodular lattice containing $P$ as a partial subalgebra has and can be done outside of $L_{0}$. It uses the coatom extension for ortholattices.

In order to prove that the free orthomodular word problem is solvable, it is sufficient, by [1], to prove that every finite "partial" orthomodular lattice $S$ can be embedded in a finite orthomodular lattice. We can assume that $S$ is a finite suborthoposet of the free orthomodular lattice $L_{0}$ on countably many generators and we shall prove that there exists a finite suborthoposet $M(S)$ of $L_{0}$ which is generated by $S$ and $M(S)$ is, with the induced structure, an orthomodular lattice (Theorem 4).

Received 12 November 1985. I thank M. Dicht1, who worked for three years on this problem, for numerous discussions. I am sorry that it was not he, but $I$, who found a solution.

Copyright Clearance Centre, Inc. Serial-fee code: 0004-9727/86 $\$ A 2.00+0.00$. 
Theorem 4 holds for all m-generated suborthoposets of $L_{0}$ with $m \leq 2$ since the free orthomodular lattice on two generators is finite $[2 ; 3.9]$. Our inductive hypothesis is:

(A) For every $m$-generated suborthoposet $T \subseteq L_{0}$ with $m<n$ there exists a finite orthomodular lattice $M(T)$ such that

(i) $T$ is a generating suborthoposet of $M(T)$,

(ii) joins and meets in $L_{0}$ of elements in $T$, which exist in $T$, are preserved in $M(T)$,

(iii) $M(T)$ can be embedded into $L_{O}$ such that $T$ is mapped identically onto itself and every chain $E \subseteq T$ generates the Boolean subalgebra $\Gamma E \subseteq L_{0}$ in $M(T)$.

Here $\Gamma A$ is, for $A \subseteq L_{0}$, the subalgebra of $L_{0}$ generated by $A$. For condition (iii) we observe that, in general, the condition on $L_{0}$ implies that, for any finite orthomodular lattice $N$ generated by $T$, there exists an isomorphic copy of $N$ in $L_{0}$. We shall write $x^{\circ}=x$ and $x^{1}=x^{\prime}$ for elements $x$ of an ortholattice.

LEMMA 1. Let $L$ be a finite ortholattice and let $a \in L-\{0,1\}$ be such that:

(i) $M=L-\left\{a, a^{1}\right\}$, with the induced stmucture, is an orthomodular Zattice

(ii) there exist $b_{0}, b_{1} \in L$ such that

$$
\left[0, a^{\varepsilon}\right)=\left[0, b_{\varepsilon}\right] \subseteq L \text { for } \varepsilon=0,1 \text {. }
$$

Then there exist a finite orthomodular lattice $N$ containing $M$ as a subalgebra and $L$ as a generating suborthoposet.

Proof. By $[2 ; p .310]$ we construct for the quasi-ideal $D=\left[0, b_{0} \vee b_{1}\right] \underline{C}^{\prime} M$ a coatom-extension $N=M \cup\left(D \times\left\{c^{\prime}\right\}\right) \cup\left(D^{\prime} \times\{c\}\right)$ where $(1, c)$ is a new coatom above the elements of $D$. If we identify $a^{\varepsilon} \in L$ with $\left(b_{1}^{1-\varepsilon}, c^{\varepsilon}\right)$ then $M$ is a subalgebra of $N$ and $L$ is embedded as a generating suborthoposet in $N$. 
LEMMA 2. Let $S$ be a finite n-generated suborthoposet of $L_{0}$ with an element $a \in S$ such that $T=S-\left\{a, a^{\prime}\right\}$ is $(n-1)$-generated. Then there exists a finite orthomoduzar zatice $M(S)$ such that (i)-(iii) of (A) hold for $S \subseteq M(S)$.

Proof. By the inductive hypothesis there exists a suborthoposet $M(T)$ of $L_{0}$ with the properties $(i)-(i i i)$ of $(A)$ for $T \subseteq M(T)$. In $T$ there exist finitely many elements $a_{1}, \ldots, a_{r}<a$ and $d_{1}, \ldots, d_{t}>a$. Define $b_{0}=a_{1} \vee \ldots \vee a_{r}$ and $b_{1}^{\prime}=d_{1} \wedge \ldots \wedge d t$. We can assume $b_{\sigma}, b_{1} \in M(T)$ since $T \cup\left\{b_{0}, b_{1}, b_{0}^{\prime}, b_{1}^{\prime}\right\}$ is $(n-1)$-generated. By 1 . there exists a finite orthomodular lattice $N=M(S)$ containing the ortholattice $M(T) \cup\left\{a, a^{\prime}\right\}$, -with $x \leqq a \leqq y$ for $x, y \in M(T)$ iff $x \leqq b_{0}$ and $b_{1}^{\prime} \leqq y_{1}-$ as a generating suborthoposet and $M(T)$, as a subalgebra. The definition of $N$ and the properties of $M(T), S$ and $L_{0}$ imply that $(i)-(i i i)$ of (A) holds for $S \subseteq M(S)$.

It follows from Lemma 2 that we can make the new inductive assumption: (B) For every $n$-generated suborthoposet $S \subseteq L_{0}$ which contains an (n-1)-generated suborthoposet $T$ with $2 \leq|S-T|<2 r$ there exists a finite orthomodular lattice $M(S)$ such that (i)-(iii) of (A) holds for $S \subseteq M(S)$.

Let $S \subseteq L_{0}$ be an $n$-generated suborthoposet with an $(n-1)$-generated suborthoposet $T$ such that $|S-T|=2 r$. Let $a \in S-T$ be such that $S$ is generated by $T \cup\{a\}$. We measure the length of an element in $S$ in terms of the generating set $D$ and choose an element $b \in S-T$ of maximal length. Then $E=S-\left\{b, b^{\prime}\right\}$ is a suborthoposet of $L_{0}$ generated by $D$ for which the inductive hypothesis (B) applies. Let $L_{1}$ be a finite orthomodular lattice which is embedded into $L_{0}$ such that (i)-(iii) hold for $E \subseteq M(E)=L_{1}$. We can assume that $b \notin L_{1}$ in the following lemma since otherwise $M(E)=M(S)$ satisfies $(i)-(i i i)$ of (B) for $S \subseteq M(S)$.

LEMMA 3. Assume that whenever $z \in E$ covers $b$, and $b$ covers two elements $x, y \in E$, then $u<b<v$ for $u, v \in E$ implies $z \leqq v$ and $u \leqq x$ or $u \leqq y$. Then there exists a finite orthomodular lattice 
$N=M(S)$, containing $L_{1} \cup\left\{b, b^{\prime}\right\}$ as a suborthoposet, such that (i)(iii) of (A) holds for $S \subseteq M(S)$.

Proof. We assume $b \notin L_{1}$. In particular, by (iii), $x \notin y^{\prime}$. In $L_{1}$ we consider the interval $[x \wedge y, z]_{1}$ and its extension to the orthoposet $N_{1}=[x \wedge y, z]_{1} \cup\left\{b, b^{\prime}\right\}$ such that $[0, b) \cap N_{1}=$ $=([0, x] \cup[0, y]) \cap N_{1}$ is a quasi-ideal $A$ in $N_{1} \cap L_{1}$ and where $a<b<c$ in $N_{1}$ implies $c=z$ and $a \leqq x$ or $a \leqq y$. By using the coatom-extension for the new coatom $b$ and the quasi-ideal $A$ there exists a finite orthomodular lattice $N_{2}$ containing $N_{1}$ as a generating suborthoposet. We can assume that $N_{2}$ is embedded in $L_{0}$ and we replace in $L_{1}$ the interval $\left[0, z \wedge\left(x^{\prime} \vee y^{\prime}\right)\right]_{1}$ by an isomorphic copy $N_{3}$ of $N_{2}$. In the product $L_{2}$ of $\left[0, z^{\prime} \vee(x \wedge y)\right]_{1} \subseteq L_{1}$ with $N_{3}$ we identify the element $\left(x \wedge y, b \wedge\left(x^{\prime} \vee y^{\prime}\right)\right)^{\varepsilon}$ with $b^{\varepsilon}$. We paste the ortholattices $L_{2}$ and $L_{3}=L_{1}-\left(\left[0, z \wedge\left(x^{\prime} \vee y^{\prime}\right)\right]_{1} \cup\left[z^{\prime} \vee(x \wedge y), 1\right]_{1}\right)$ to the ortholattice $N$ along the common segment $\left[0, z^{\prime} \vee(x \wedge y)\right]_{1} \times\{0,1\}$. A new element in $N-L_{1}$ is only comparable with an element $x \in L_{3}$ if $x \in L_{2}$ holds. Therefore if, for $c, d \in L_{3}$, we have both $c<d^{\prime}$ and $c^{\prime} \wedge d^{\prime} \notin L_{3}$, then $c^{\prime} \wedge d^{\prime}<z \wedge\left(x^{\prime} \vee y^{\prime}\right)$ and we extend the partial order on $N$ for these elements and their orthocomplements by $u \leqq c^{\prime}$, $d^{\prime}$ for all $u \leqq c^{\prime} \wedge d^{\prime}$ in $N$. This way we obtain from $N$ an orthomodular lattice $M(S)$ such that (i)-(iii) of (B) holds for $S \subseteq M(S)$.

Some remarks additional to Lemma 3 are: If there exists $A \subseteq S$ with $|A| \geqq 2$ such that $z$ covers $b$ and $b$ covers $a$ for all $a \in A$ and $\dot{z} \in S$, and if $u<b<v$ for $u, v \in E$ implies $z \leq v$ and $u \leqq a$ for some $a \in A$, then we can use the same arguments for the quasiideal $\cup\{[0, a] \mid a \in A\} \cap N_{1}$ replacing $([0, x] \cup[0, y]) \cap N_{1}$ in Lemma 3 . We also observe that, for the case where two elements $u, v$ (or more) cover $b$ and $b$ covers two elements $x, y$ (or more) in $S$ such that $a<b<c$ for $a, b \in S$ implies $a \leqq x$ or $a \leqq y$ and $u \leqq c$ or $v \leqq c$, there exists an element $z$ in $L_{1}$ such that for the $E$-generated 
element $b$ either $b<z \leqq u, v$ or $x, y \leqq z<b$ holds. In the first case we apply the construction of Lemma 3 . to the interval $N_{1}=[x \wedge y, z]_{1} \subseteq L_{1}$. The other case is dual and for the case where more than two elements in $S$ cover $b$ or are covered by $b$ we apply the procedure just described (several times if necessary). We conclude that the assertion of Lemma 3 holds without the additional assumptions on $E$.

THEOREM 4. Let $S \subseteq L_{0}$ be a finite suborthoposet. Then there exists a finite suborthoposet $M(S) \subseteq L_{0}$ containing $S$ as a generating set which, together with its induced structure, is on orthomoduzar zattice.

\title{
References
}

[1] T. Evans, "Embeddability and the word problem," J. London Math. Soc. 28 (1953), 76-80.

[2] G. Kalmbach, Orthomodular Zattices. (Academic Press, London, 1983).

\author{
Abt.Math.III, O.E. \\ University $\mathrm{U} 1 \mathrm{~m}$ \\ D-7900 ULM
}

West Germany. 